\title{
Recent Advances in Superconductivity and Vortex Matter: Selected Topics
}

\author{
Vyacheslav R. Misko \\ Theory of Quantum and Complex Systems (TQC) \\ Department of Physics, University of Antwerp \\ Universiteitsplein 1, B-2610 Antwerpen, Belgium \\ vyacheslav.misko@uantwerpen.be
}

Received 29 July 2017

Accepted 29 September 2017

Published 28 December 2017

\begin{abstract}
The more-than-a-century history of superconductivity is marked by a great deal of progress in the understanding of this fascinating phenomenon as well as discovery and design of new superconducting materials and systems with enhanced critical parameters. Despite the achievements in theory, experiment and technology, many questions still remain open related to the mechanisms of superconductivity in so-called unconventional materials and aspects related to applications of superconductors which stimulates continuous research interest in the field. In this short review paper, we discuss some important recent advances in superconductivity and vortex matter made over about the last decade. In particular, we focus on novel iron-based and multigap superconductors and vortex matter in multi-component systems including multiband superconductors and multi-component Bose-Einstein condensates. We also discuss recent advances in manipulating vortex matter in nano-engineered superconducting systems such as non-periodic pinning arrays which can be used as devices that enhance the critical superconducting parameters and thus open new possibilities for applications of superconductors.
\end{abstract}

Keywords: Iron-based superconductors; multigap superconductors; multi-component condensates; nano-engineered supercondutors; vortex matter; vortex pinning; superconducting critical parameters.

\section{Novel Superconductors}

Superconductivity was first observed by Kamerlingh Onnes in Leiden in $1911 .^{1}$ The transition to the superconducting state occurs in many metals and alloys when their electrical resistivity drops to zero at a sufficiently low temperature, called superconducting critical temperature $T_{c}$. Since then, a great deal of progress has been made in the understanding of the phenomenon of superconductivity. A phenomenological theory based on the Maxwell equations of classical electrodynamics was

This is an Open Access article published by World Scientific Publishing Company. It is distributed under the terms of the Creative Commons Attribution 4.0 (CC-BY) License. Further distribution of this work is permitted, provided the original work is properly cited. 
first proposed by London and London in $1935 .{ }^{2}$ The London equation, in particular, describes the Meissner effect, i.e., the expulsion of the magnetic field from a superconductor. Extending the Landau theory of phase transitions to superconductors, Ginzburg and Landau proposed in 1950 a set of equations, known as GinzburgLandau equations. ${ }^{3}$ The Ginzburg-Landau phenomenological theory explains many phenomena in superconductivity and has been successfully used for the description of, e.g., effects observed in mesoscopic and nano-structured superconductors such as magnetic flux penetration, flux pinning, etc. In 1957, the quantum theory of superconductivity (BSC theory) was proposed by Bardeen et al. The BSC theory has a very broad applicability ranging from $\mathrm{He}^{3}$ atoms in the condensed phase, to type I and type II superconductors, and to high-temperature superconductors. Despite its more-than-a-hundred-years history, superconductivity is actively developing field. Recent discoveries of cuprate and iron-based superconductors (IBSC) not only advanced the state-of-the-art in terms of the unique superconducting properties (such as reaching high critical temperatures $T_{c}$ ) and thus enhanced the potential for practical applications, but also raised important fundamental questions on new mechanisms of superconductivity.

IBSC are a new class of materials that can be referred to as "novel superconductors". First discovered just about 10 years ago, these materials demonstrated a significant increase of both in number of various IBSC compounds and unique properties, e.g., the superconducting critical temperature, $T_{c}$. The first discovered IBSC compound was LaFePO ${ }^{4}$ with $T_{c}=4 \mathrm{~K}$. Subsequently, superconductivity was found in $\mathrm{LaNiAsO}^{5}$ with $T_{c}=2.44 \mathrm{~K}$ in 2007 , and then $T_{c}$ showed a strong jump up to $26 \mathrm{~K}$ in early 2008 for $\mathrm{LaFeAsO}_{1-x} \mathrm{~F}_{x}{ }^{6}$

The electromagnetic properties of $3 \mathrm{~d}$ transition metal (TM) oxypnictides vary drastically with TM. ${ }^{7,8}$ The early attempts of synthesis TMs and $\mathrm{Cu}$ oxypnictides were unsuccessful, even using high pressure up to $9 \mathrm{GPa}$, and no distinct correlation was found between the stability of the compound and the kind of TM. Bulk superconductivity was detected in compounds with $\mathrm{TM}=\mathrm{Fe}^{2+}$ and $\mathrm{Ni}^{2+}$, both having even number of $3 \mathrm{~d}$ electrons, but no superconductivity has been found in materials with $\mathrm{TM}=\mathrm{Cr}^{2+9}$ with $3 \mathrm{~d} 4$ electronic configuration. Undoped LaFeAsO is an antiferromagnetic metal but does not exhibit superconductivity. For $\mathrm{TM}=\mathrm{Mn}$, an exceptionally high electron doping is possible by applying $\mathrm{H}^{-10}$ instead of $\mathrm{F}^{-}$as a substituent for the oxygen site, and the transition of antiferromagnetic insulator to ferromagnetic metal was observed but no $T_{c}$ appeared. For the compound LaCoAsO, which is an itinerant ferromagnetic metal, ${ }^{11}$ no $T_{c}$ exceeding $10 \mathrm{~K}$ has been reported in the 1111 system up to date, with the exception for the iron oxyarsenides. Recently, the critical temperature up to $T_{c}=54 \mathrm{~K}$ and $56 \mathrm{~K}$ has been reported in IBSC compounds $\mathrm{SmFeAsO}_{1-x} \mathrm{H}_{x}$ and $\mathrm{NdFeAsO}_{1-x} \mathrm{H}_{x}$ synthesized using high pressure technique. ${ }^{12} \mathrm{~A}$ detailed discussion on the various IBSC materials, their structure and properties can be found in the recent review. ${ }^{13}$

IBSC are another member of the high temperature superconductors (HTSs), after cuprate superconductors. Although IBSC share some similarities with cuprate 
superconductors, such as a layered structure, very high upper critical fields, and a doping phase diagram, important differences exist between the two families. Cuprates are doped Mott insulators with strong correlation and single-band behavior, while IBSC are metallic with multiband electronic structures. ${ }^{14}$ The existence of disconnected Fermi surfaces with electron and hole characters, and spin or orbital fluctuations are supposed to be responsible for the high value of $T_{c}=54 \mathrm{~K}$ in IBSC based on either the $s_{ \pm}{ }^{15}$ or $s_{++}$scenario. ${ }^{16}$ The nesting between electron and hole bands is supposed to be related to the high value of $T_{c}$ in IBSC based on the scenario of $s_{ \pm}$-pairing. This multiband feature also influences the normal state transport properties of IBSC. Strong temperature dependent Hall coefficients, large magnetoresistance (MR), and a linear temperature dependence of resistivity were observed, especially in iron pnictides. ${ }^{17-20}$ Moreover, a Dirac cone state, coming from the nodes of the spin-density-wave (SDW) gap by complex zone folding in different bands, was observed in $\mathrm{Ba} / \mathrm{SrFe}_{2} \mathrm{As}_{2}$ and $\mathrm{La} / \mathrm{PrFeAsO} .{ }^{21-23}$ Although the weight of the Dirac cone state is small, it can dominate the transport properties because of its extremely high mobility. As a consequence, a large and linear temperature-dependent MR was observed. In addition, when interpreting experimental data, a possibility of phase separation should be kept in mind. ${ }^{24}$

In the family of IBSC, iron chalcogenide $\mathrm{Fe}_{1+y} \mathrm{Te}_{1-x} \mathrm{Se}_{x}$ attracted much attention due to its simple structure, which is convenient to probe the superconducting mechanism. In addition, its less toxic nature is also advantageous to application in IBSC. Band structure calculations and angle-resolved photoemission spectroscopy (ARPES) prove the multiband structure in $\mathrm{Fe}_{1+y}(\mathrm{Te} / \mathrm{Se}),{ }^{25-27}$ which is characterized by hole bands around the $\Gamma$ point and electron bands around the $M$ point, similar to iron pnictides. The transport features that are characteristic of a multiband structure, such as a large magnetoresistance, have been recently reported. ${ }^{27} \mathrm{~A}$ nonlinear Hall resistivity was observed in a fully annealed crystal, and the magnetoresistance was drastically enhanced after annealing, which proves the multiband nature of $\mathrm{Fe}_{1+y} \mathrm{Te}_{0.6} \mathrm{Se}_{0.4}$. In addition, a temperature-dependent linear MR was observed in the annealed crystal, which was interpreted by the possible existence of Dirac fermions. ${ }^{28}$

\section{Multicomponent Superconductivity}

Multicomponent superconductivity is realized in multiband superconductors when an interband pairing interaction is considerably weaker than the intraband interactions (see, e.g., Ref. 29). In a multiband superconductor, each band is considered to have a condensate with an amplitude and phase that weakly interacts with the condensates of the other bands. The system has multiple quantum phases, which is not a straightforward extension of a conventional single-band superconductor with only one quantum phase.

The first attempt to tackle multiband superconductors was made by Suhl et al. by deriving a two-band extension of the BSC theory, ${ }^{30}$ two years after the original microscopic BCS theory of superconductivity (valid for an isotropic single superconducting 
gap) was formulated by Bardeen et al. ${ }^{31}$ Two different magnitudes were assigned to two different superconducting gaps, whereas the superconducting phase was assumed to be identical for the two gaps. Further steps to improve the model were made by Kondo ${ }^{32}$ and Peretti ${ }^{33}$ who independently introduced different phases for different gaps, with a fixed difference of $\pi$ radians. As a collective excitation potentially present in the transition metal superconductors, Leggett ${ }^{34}$ introduced a phase difference other than 0 or $\pi$. This small fluctuation in the phase difference is now called as a "Leggett mode". In 2001, Tanaka extended this fluctuation to $2 \pi,{ }^{35,36}$ yielding an interband phase-difference soliton ( $i$-soliton). The very weak interband interaction discovered in the multi-layer cuprate superconductor $\mathrm{Cu}-1234$ was a trigger for this finding.

Among the materials considered as candidates to realize the multicomponent superconductivity based on multiband superconductors, the transition metals, such as $\mathrm{Nb}$ and $\mathrm{V}$, were first predicted to have a multiband nature. The Leggett mode was theoretically discovered for these materials. ${ }^{34} \mathrm{In}$ a similar way, $\mathrm{NbSe}_{2}$, graphite intercalation compounds, and the Chevrel compound were investigated and revealed multiband properties. Multilayer cuprate superconductors such as $\mathrm{CuBa}_{2} \mathrm{Ca}_{3} \mathrm{Cu}_{4} \mathrm{O}_{y}$ $(\mathrm{Cu}-1234)$, which is obtained by substituting $\mathrm{Ca}-\mathrm{CuO}_{2}-\mathrm{Ca}$ for the $\mathrm{Y}$ in $\mathrm{YBa}_{2} \mathrm{Cu}_{3} \mathrm{O}_{y}$ (Y-123) and has four $\mathrm{CuO}_{2}$ planes in one unit cell, exhibited the multiband nature. ${ }^{37,38}$ It was experimentally suggested that the interband interaction in these materials is two orders of magnitude smaller than the intraband interaction. ${ }^{39} \mathrm{Cu}-$ 1234 was the first material satisfying the Leggett condition, whereby the interband interaction was far smaller than the intraband interaction. Consideration of the basic properties of $\mathrm{Cu}-1234$ suggested the interband phase difference soliton ( $i$-soliton). ${ }^{34,35}$ Indeed, there was an experimental indication of the formation of the vortex molecule composed of the fractional vortex and interband phase soliton in $\mathrm{CuBa}_{2} \mathrm{Ca}_{2} \mathrm{Cu}_{3} \mathrm{O}_{y}$ $(\mathrm{Cu}-1223)$, which is a member of the family of $\mathrm{Cu}-1234 .{ }^{40}$ Recently studied $\mathrm{MgB}_{2}{ }^{41}$ and pnictidescite ${ }^{4}$ are also considered as attractive candidates. The artificial structures, such as a thin $\mathrm{Al} / \mathrm{AlO}_{x} / \mathrm{Al}$ multilayer, is the most promising platform for the study of the $i$-soliton. ${ }^{42}$ When the multilayer is much thinner than the magnetic penetration depth, it is considered to mimic the multiband superconductor. The direct observation of the $i$-soliton ${ }^{43}$ and fractional vortices, ${ }^{43,44}$ which constitute symbolic topology discussed in the multicomponent superconductivity, were reported using the multilayer system.

\section{Vortex Matter in Multiband Superconductors}

Bands of a multiband superconductor are characterized, in general, by different characteristic lengthscales, the coherence length, $\xi_{i}$, and the magnetic field penetration depth, $\lambda_{i}$. As a result, different bands have different Ginzburg-Landau (GL) parameters $\kappa_{i}=\lambda_{i} / \xi_{i}$ that define the lengthscales of the vortex-vortex interaction. On the other hand, it is known from the theory (see, e.g., Ref. 45) that in the case of multiscale vortex-vortex interactions, two or more purely repulsive potentials 
characterized by different lengthscales, can result in the appearance of an attractive component in the intervortex interaction. Therefore, this idea can be explored as a simple interpretation of the origin of an attractive interaction in two- and multiband superconductors. ${ }^{46-50}$ Furthermore, in the special case of a two-band superconductor (called "type-1.5 superconductor" ${ }^{47}$ ), like $\mathrm{MgB}_{2}$, two different GL parameters, corresponding to the different bands, can be either smaller or larger than the dual point, $\kappa_{\pi}<1 / \sqrt{2}$ (type-I) and $\kappa_{\sigma}>1 / \sqrt{2}$ (type-II), thus leading straightforward to a nonmonotonic repulsive-attractive intervortex interaction. ${ }^{46-48}$ In addition, the appearance of an effective attractive term has been recently demonstrated also for nonpairwise vortex-vortex interaction. ${ }^{51}$

The repulsive-attractive interaction, while being less common and less studied in the case of vortices, is known in physics, e.g., in colloids. Thus, systems interacting via repulsive-attractive potential (in particular, of the Lennard-Jones type) were extensively studied in physics and were shown to result in a variety of non-trivial patterns, including stripes, labyrinths, lattices with voids, etc. ${ }^{52,53}$ For vortices interacting via non-monotonic repulsive-attractive interactions, vortex pattern formation has been analyzed, ${ }^{45,51,54-60}$ using various models. In particular, vortex clusters, stripes, labyrinths, deformed lattices, and lattices with voids were found. ${ }^{59}$ In addition, systems with non-monotonic interaction were shown to display unusual dynamics, such as size-selective dynamical cluster formation and re-orientation of longitudinal stripes to transverse stripes. ${ }^{60}$ Some of the obtained static patterns were employed to explain the observed vortex patterns in superconductors, either in twoband materials, such as $\mathrm{MgB}_{2}$, or in low- $\kappa$ superconductors.

The numerical simulations using model repulsive-attractive interactions between vortices fairly reproduced such observed features as the formation of vortex clusters and vortex stripes, i.e., the most generic types of patterns derived from non-monotonic interactions. ${ }^{52,53}$ However, as recently observed, ${ }^{61}$ despite these basic similarities, the measured vortex patterns are much less perfect: e.g., less ordered broken stripes $^{47,48,50}$ or chains of dimers rather than regular stripes. ${ }^{62}$ Based on a comparison of the calculated regular vortex patterns (see, e.g., Refs. 45, 51, 59, 60, 63) and the experimentally observed vortex patterns in multiband superconductors reported in the literature, ${ }^{47,48,50}$ Zhao et al. ${ }^{61}$ proposed to improve the model intervortex interaction by including the effects resulting from vortex-core deformations. Namely, they analyzed effects related to a short-range effective attraction in the overall repulsive intervortex interaction, in presence of random pinning, and frustration in the vortexvortex interaction, combined with random pinning. ${ }^{61}$ The appearance of frustration in the intervortex interaction can be understood from the fact that, when deformed, vortex cores elongate in the direction of the closest neighbor resulting in an anisotropy and thus breaking the symmetry of the interaction in the system. ${ }^{64,65}$ As a result, the interaction of the vortex with elongated core with the next closest neighbor depends on the orientation of the vortex core with respect to the direction to that second closest neighbor. Using the improved model, a rich variety of phases (or "morphologies") was revealed,${ }^{61}$ for increasing vortex density, including: 


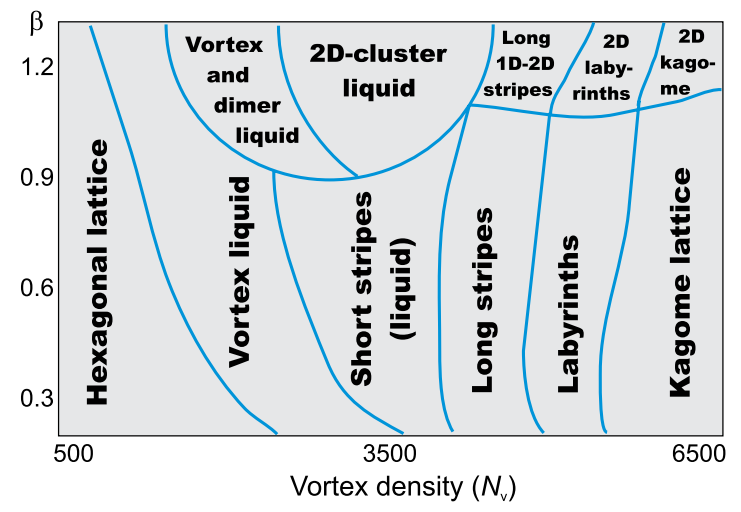

Fig. 1. A diagram showing the various morphologies of the vortex patterns that are encountered when varying the intervortex attraction force strength $\beta$ and the vortex density $N_{v}$. For more details and a morphology diagram in the plane "pinning strength $f_{p}$ — vortex density $N_{v}$, see Ref. 61.

(i) a hexagonal vortex lattice, (ii) a liquid of vortex dimers, (iii) short stripes, (iv) long stripes, (v) interconnected stripes and labyrinths, and (vi) kagomé lattices (see Fig. 1). Adding random pinning of increasing strength results in the formation of patterns which are a mixture of short branching stripes and individual vortices or vortex dimers. Due to frustration in the intervortex interaction, for high enough vortex densities, the resulting vortex patterns represent a disordered mixture of vortex dimers and four-vortex chains, with a small fraction of single vortices and three-vortex chains. ${ }^{61}$ The simulated complex vortex patterns are in agreement with the recently observed vortex patterns in $\mathrm{MgB}_{2}$ films. ${ }^{50,62}$

\section{Composite Vortices in Multi-Component Bose-Einstein Condensates}

The discovery of superconductors with multiple superconducting gaps provided new prospects for the study of vortices. Magnesium diboride, $\mathrm{MgB}_{2}$, discussed in the previous section, is an example of a two-gap superconductor where one gap corresponds to much more strongly bound pairs than the other gap. ${ }^{47,48} \mathrm{In} \mathrm{MgB}_{2}$, vortices have been observed to form stripe patterns, which where not due to pinning centers, defects or edge effects. ${ }^{50}$ This was an indication that the interactions between the vortices may have a dipolar component, due to the composite nature of vortices in these materials. The precise interpretation of the observed patterns in these materials with two superconducting components is still under discussion. ${ }^{47,48,50,61,62}$

Recent advances in ultracold atom physics have allowed for an unprecedented level of control over the experimental parameters of ultracold atomic gases. The number of particles, temperature, confinement geometry, dimensionality and interaction strength can be tuned accurately over a broad range of values. Due to this experimental flexibility, ultracold atoms are also known as a quantum simulator for many-body systems and theories. ${ }^{66}$ Motivated by the recent observations of composite vortices in two-gap superconductors, Tempere et al. ${ }^{67}$ investigated the 
occurrence and stability of composite vortices in a two-component Bose-Einstein condensate (BEC). The interaction between two composite vortices was analyzed and shown to consist of three various contributions: two strong, long-range repulsive contributions (corresponding to the intra-component vortex interaction) and one weak, short-range attractive contribution for the inter-component interaction, due to the overlap of the component order parameters. ${ }^{67}$ It was shown that vortices in the two-components of the BEC mixture can overlap and form a composite vortex object, as a function of the inter-component interaction strength. However, when two such composite vortices approach each other, their mutual interaction may cause the composite object to dissociate in its component vortices. The resulting phase diagram revealed two distinct areas: a first where the composite vortices dissociate and a second where they remain stable despite their offset cores. It was found that for a sufficiently strong inter-component interaction, the system will be stable with respect to dissociation at all distance scales.

\section{Vortex Matter in Non-Periodic Pinning Arrays}

\subsection{Enhancement of the critical current in quasiperiodic pinning arrays}

Since the discovery of Abrikosov vortices in type II superconductors, their pinning (i.e., immobilization) has been and still remain a topic of considerable interest, both with respect to the fundamental physical properties of vortex matter and with respect to device applications. In the case of device applications, for bulk or thick film superconductors, the inclusion of nanodefects, acting as pinning sites, typically randomly distributed within the superconductors, has been shown to significantly enhance the critical current density, which is important for increasing the currentcarrying capacity of wires and tapes, e.g., for applications in superconducting magnets (see, e.g., Ref. 68 and references therein).

Recent progress in the fabrication of nanostructures has provided a wide variety of well-controlled lithographically defined artificial pinning sites, and experiments can control both the location and the strength of each pinning center in vortexconfining Arrays of Pinning Sites (APS) (see, e.g., Refs. 69-72). In this context, it has been shown that, e.g., microholes (antidots) or magnetic dots may improve the performance of micro-electronic thin-film devices. Examples are the suppression of low-frequency flux noise in superconducting quantum interference devices (SQUIDs) by strategically positioned antidots ${ }^{73,74}$ or the enhancement of the quality factor of superconducting microwave resonators operated in magnetic fields, which can be achieved by incorporating antidot arrays into the thin film structures. ${ }^{75}$ Highly sensitive high- $T_{c}$ superconducting SQUID-based magnetic field sensors are used in medical tomography. Immobilization of undesired moving vortices in the active area of SQUIDs substantially enhances the sensitivity of the sensor.

From a basic point of view, it is interesting to explore the optimum pinning landscape provided by APS. One convenient way to do this is to investigate the 


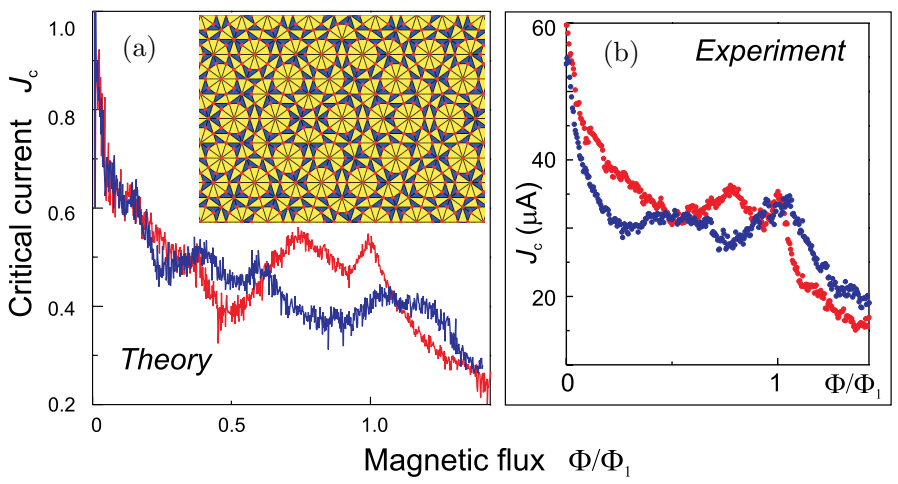

Fig. 2. Normalized critical current (experiment and theory), $J_{c}$ versus normalized magnetic flux, $\Phi / \Phi_{1}$ ( $\propto$ number of vortices $N_{v}$ ), for QP Tuebingen tiling. The function $J_{c}\left(\Phi / \Phi_{1}\right)$ for a five-fold Penrose-tiling APS is shown by red (gray) line, for comparison. The comparison shows that, while a five-fold Penrosetiling APS provides a broad maximum for fluxes $\Phi<\Phi_{1}$, in the case of a QP Tuebingen-tiling APS, the enhancement of the critical current is observed for magnetic fluxes $\Phi>\Phi_{1} \cdot{ }^{86}$ For a detailed discussion and more results on other QP APS, see Refs. 76, 77, 86.

critical current $J_{c}$ versus magnetic flux $\Phi$ for different arrangements of pinning sites. Periodic artificial APS were first proposed as devices able of trapping magnetic flux and thus enhancing $J_{c}$. However, the efficiency of periodic APS is strongly limited to so-called matching fields, i.e., when the number of vortices is commensurate with the number of pins, thus resulting in strong but very narrow peaks in the critical current versus magnetic flux, $J_{c}(\Phi)$. As it was shown first theoretically, ${ }^{76,77}$ and then verified experimentally, ${ }^{78-81}$ this shortcoming can be overcome by using more sophisticated pinning topologies, e.g., quasiperiodic (QP) APS. In particular, using a five-fold Penrose tiling (see, e.g., Refs. 82-85) as an APS (i.e., placing pins on the vertices of a Penrose tiling) results in a considerable enhancement of $J_{c}(\Phi)$, i.e., significant broadening of the peak in $J_{c}(\Phi)$, as was recently demonstrated theoretically ${ }^{76,77}$ and experimentally. ${ }^{79,80}$ The underlying idea of using QP APS is that, contrary to regular APS, they include many periodicities and thus can trap vortices for magnetic fluxes other than matching values $\Phi_{i}$.

The counter-intuitive mechanism of flux pinning by QP APS could be understood as follows. On the one hand, an Abrikosov hexagonal vortex lattice is incommensurate with a QP APS for any vortex densities which would imply that QP APS could not be used for magnetic flux pinning for any flux density, provided the vortex lattice is rigid. However, the vortex lattice is elastic. Thus, this is the elasticity of the vortex lattice, on the other hand, that provides pinning of the vortex lattice by a QP APS, in a broad range of vortex densities. Small elastic deformations of the vortex lattice, together with many periodicities of a QP APS, result in a striking pinning properties of QP pinning devices. ${ }^{76,77}$

The unusual pinning properties of QP APS have been further investigated, both numerically and experimentally, ${ }^{86,87}$ for other QP tilings than a five-fold Penrose tiling, e.g., for QP nonpisot Penrose tiling, square-triangle and square-triangle 
nonpisot tiling, Goodman-Strauss tiling, Watanabe-Ito-Soma tiling, Tuebingen tiling (see Fig. 2), and shield tiling. ${ }^{86}$ The essential difference in the flux pinning for magnetic fluxes below and above the matching value $\Phi=\Phi_{1}$ has been discussed and it was demonstrated, theoretically and experimentally, how the critical current $J_{c}(\Phi)$ can be improved in the important regime where $\Phi>\Phi_{1}$, by using novel artificial APS. ${ }^{86}$

\subsection{Magnetic flux pinning in hyperbolic-tessellation and other graded-density pinning arrays}

Non-Euclidean geometries have had a profound effect in physics ${ }^{88}$; not only in general relativity, but also in condensed matter physics. ${ }^{89-91}$ Some examples include order and defects in liquids and metallic glasses, ${ }^{92}$ polytope models of glass ${ }^{93}$ in a curved icosahedral space, icosahedral bond-orientational-order in supercooled liquids, ${ }^{94}$ etc. Topological defects, i.e., disclinations, dislocations and vortices, were studied on rigid substrates of spatially-varying Gaussian curvature. ${ }^{95}$

The geometry of a mesoscopic superconductor and the underlying pinning have a strong impact on its vortex pattern. For example, the appearance of vortex concentric "shells" ${ }^{96-98}$ in mesoscopic disks, which can even merge into so-called giant vortices ${ }^{99}$ in very small disks. In symmetric polygons, e.g., triangles and squares, vortices tend to form patterns with the symmetry consistent with the polygon boundary. Moreover, the sample symmetry can even lead to a spontaneous generation of antivortices to restore the broken symmetry for incommensurate magnetic flux. ${ }^{100,101}$ Furthermore, vortex patterns can also be produced by various APS (see, e.g., Ref. 76)

Using APS incommensurate with vortex lattices results in an elastic deformation of the vortex lattice and thus in an increase in the elastic energy. However, pinning properties of a superconductor can be even improved by using incommensurate APS sites, as recently demonstrated (theoretically ${ }^{76,77}$ and experimentally ${ }^{79,80,86}$ ) for QPtiling APS (see the previous section). The important property of QP APS $76,77,79-81,86$ is the existence of many built-in periods resulting in flux pinning for various flux densities. This unique property, in turn, opens the possibility for the design of fluxonics devices with enhanced pinning over a broad range of fields.

One realization of such fluxonic devices has been recently proposed that uses a superconductor with pinning sites placed on the vertices of a hyperbolic tessellation (HT). ${ }^{102}$ In their analysis, the authors ${ }^{102}$ used a conformal projection of an infinite regular tiling from a hyperbolic space to a finite-size disk in a 2D Euclidean space, i.e., a Poincaré disk representation. As a result, this model showed some similarities to mesoscopic symmetric experimental samples, ${ }^{96,97,99}$ although with additional internal structure and less rigid "boundaries". HTs are obtained as a conformal projection $^{\mathrm{a}}$ of a tiling of regular symmetric polygons in a hyperbolic space to a twodimensional (2D) Euclidean space (similarly, QP tilings are obtained as a 2D projection

\footnotetext{
a In the literature, the tilings (such as hyperbolic tessellations) obtained as a result of a conformal projection of regular polygons, are often called "conformal crystals". Other examples include, e.g., a conformal projection of regular polygons from hyperbolic space to a 2D Euclidean semi-space.
} 

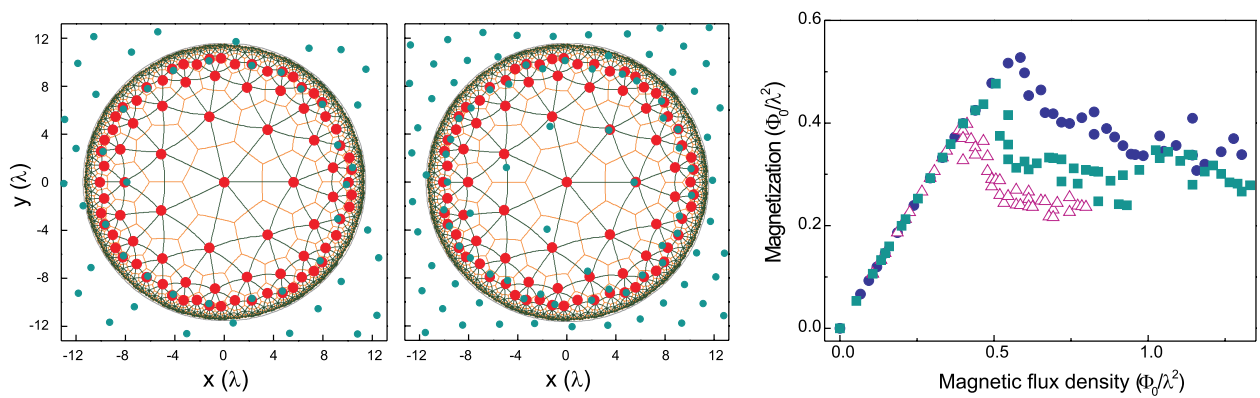

Fig. 3. Penetration of magnetic flux in a HT array of pinning sites. For relatively low fluxes (left panel), the external magnetic flux is shielded by the outer row of pinning sites giving rise to perfect diamagnetic response similar to that in a Meissner state of a superconductor. For increasing applied magnetic flux (central panel), the flux partially penetrates in the interior of the HT APS in a strongly heterogeneous way, in contrast to the conventional Bean-model scenario. The magnetization curves are shown in the right panel for varying pinning strength: $f_{p} / f_{0}=1.2$ (circles), 1.0 (squares) and 0.8 (open triangles). Note that for low values of the applied magnetic flux, the magnetization grows linearly with the flux with the slope equal to one, i.e., the interior of the device is fully screened by the outer shell of the pinning sites, similar to the Meissner effect. This effect weakens with decreasing pinning strength resulting in a weaker magnetization (as in samples without HT APS). HT APS displays a remarkable asymmetry in flux entry and exit resulting in a strong hysteresis in the the magnetization (see Ref. 102). Due to the asymmetry in flux entry and exit, this device can be used as a "flux capacitor". ${ }^{102}$

of tilings which are periodic in a higher dimensional space). As a result, a 2D HT is a set of topologically equivalent tilings of decreasing sizes from the center and towards the edge, see Fig. 3. Each tessellation is represented by a Schläfli symbol of the form $\{p, q\}$, which means that $q$ regular $p$-gons surround each vertex. There exists a HT $\{p, q\}$ for every $p, q$ such that $(p-2)(q-2)>4 .^{88-90,92,93}$ Note that the ones based on a regular $\{p, q\}$ are the same as the dual ones based on a regular $\{q, p\}$, but shown in a different orientation (shown by gray and orange lines in Fig. 3).

It was demonstrated ${ }^{102}$ that HT APS can efficiently trap flux of different densities, in contrast to periodic APS which are efficient for few specific matching flux values. Vortex matter in this device can coexist in three different phases, namely, in a liquid phase (near the center), in a viscous liquid vortex phase (further from the center), and in a solid phase (near the boundary). The analysis ${ }^{102}$ revealed that for relatively low fluxes, the outer row of pinning sited shields the interior of the sample giving rise to a strongly heterogeneous magnetic flux penetration, in contrast to that predicted by the conventional Bean model. The magnetization of this device has a linear part with the slope equal to one, which is indicative of a perfect diamagnetism similar to that in a Meissner state of a superconductor, although in a HT APS this occurs due to the shielding effect of trapped vortices in the outer pinning ring. Due to the asymmetry in flux entry and exit, the magnetization of the HT APS displays a remarkable hysteresis. ${ }^{102}$ Note that, in subsequent simulations, similar results were found for APS obtained as a conformal projection to a 2D Euclidean semi-space (see footnote a), namely, the hysteretic magnetization curves ${ }^{103,104}$ and the asymmetry in the flux entry and exit. ${ }^{105}$ 
The theoretical predictions ${ }^{102}$ were subsequently verified in experiments with graded pinning arrays. Thus, Motta et al. ${ }^{106}$ investigated a graded distribution of antidots in superconducting a- $\mathrm{Mo}_{79} \mathrm{Ge}_{21}$ thin films by means of magnetization and magneto-optical imaging measurements. The pinning landscape had maximum density at the sample border, decreasing linearly towards the center. It was shown that the overall performance of the system was noticeably superior to that for a sample with uniformly distributed antidots: For high temperatures and low fields, the critical current was enhanced ${ }^{106}$ thus confirming the relevance of graded landscapes on the enhancement of pinning efficiency. ${ }^{102}$ Wang et al. ${ }^{107}$ experimentally investigated the critical currents of MoGe superconducting films with a conformal array of nanoscale holes. They found a significant critical current enhancement at high magnetic fields. The authors ${ }^{107}$ attributed the enhanced performance to the arching effect that prevented vortex channeling occurring in samples with a regular lattice of holes. The magneto-transport properties of a conformal crystal and a randomly diluted pattern were experimentally investigated and compared with that of a triangular reference lattice. ${ }^{108}$ The authors found that, for the conformal crystal, the magnetoresistance was below the noise floor indicating highly effective vortex pinning over a wide magnetic field range. ${ }^{108}$ In a recent experiment, George et al. ${ }^{109}$ investigated the critical current density $J_{c}$ in uniform, graded and spaced arrays of $3 \mu \mathrm{m}$ triangular antidots in pulsed laser deposited $\mathrm{YBa}_{2} \mathrm{Cu}_{3} \mathrm{O}_{7}$ (YBCO) superconducting thin films. It was found that almost all types of non-uniform arrays, including graded ones enhanced $J_{c}$ over the broad applied magnetic field and temperature range due to the modified critical state, whereas uniform arrays of antidots either reduce or produce no effect on $J_{c}$ compared to the original (as deposited) thin films. ${ }^{109}$

\section{Acknowledgments}

This work was supported by the Flemish Research Foundation (FWO-Vl) and by the Research Fund of the University of Antwerp.

\section{References}

1. H. Kamerlingh Onnes, Further experiments with liquid helium. D. On the change of the electrical resistance of metals at very low temperatures. V. The disappearance of the resistance of mercury. Akad. van Wetenschappen (Amsterdam) 14 (1911) 818-821.

2. F. London and H. London, The electromagnetic equations of the supraconductor, Proc. Roy. Soc. (London) A 149 (1935) 71-88.

3. V. L. Ginzburg and L. D. Landau, On the Theory of superconductivity (In Russian), $Z h$. Eksp. Teor. Fiz. 20 (1950) 1064-1082.

4. Y. Kamihara, H. Hiramatsu, M. Hirano, R. Kawamura, H. Yanagi, T. Kamiya and H. Hosono, Iron-based layered superconductor: LaOFeP, J. Am. Chem. Soc. 128 (2006) 10012-10013. 
5. T. Watanabe, H. Yanagi, T. Kamiya, Y. Kamihara, H. Hiramatsu, M. Hirano and H. Hosono, Nickel-based oxyphosphide superconductor with a layered crystal structure, LaNiOP, Inorg. Chem. 46 (2007) 7719-7721.

6. Y. Kamihara, T. Watanabe, M. Hirano and H. Hosono, Iron-based layered superconductor $\mathrm{La}\left[\mathrm{O}_{1-x} \mathrm{~F}_{x}\right] \mathrm{FeAs}(\mathrm{x}=0.05-0.12)$ with $\mathrm{T}_{c}=26 \mathrm{~K}$, J. Am. Chem. Soc. $130(2008)$ $3296-3297$.

7. H. Hosono, Two classes of superconductors discovered in our material research: Ironbased high temperature superconductor and electride superconductor, Phys. C $\mathbf{4 6 9}$ (2009) 314-325.

8. H. Hosono and K. Kuroki, Iron-based superconductors: Current status of materials and pairing mechanism, Phys. C 514 (2015) 399-422.

9. S. W. Park, H. Mizoguchi, K. Kodama, S. Shamoto, T. Otomo, S. Matsuishi, T. Kamiya and $\mathrm{H}$. Hosono, Magnetic structure and electromagnetic properties of $\mathrm{LnCrAsO}$ with a ZrCuSiAs-type structure ( $\mathrm{Ln}=\mathrm{La}, \mathrm{Ce}, \mathrm{Pr}$, and Nd), Inorg. Chem. 52 (2013) 1336313368.

10. T. Hanna, S. Matsuishi, K. Kodama, T. Otomo, S. Shamoto and H. Hosono, From antiferromagnetic insulator to ferromagnetic metal: Effects of hydrogen substitution in LaMnAsO, Phys. Rev. B 87 (2013) 020401(R), 1-5.

11. H. Yanagi, R. Kawamura, T. Kamiya, Y. Kamihara, M. Hirano, T. Nakamura, H. Osawa and H. Hosono, Itinerant ferromagnetism in the layered crystals LaCoOX (X = P, As), Phys. Rev. B $\mathbf{7 7}$ (2008) 224431, 1-7.

12. H. Hanna, Y. Muraba, S. Matsuishi, N. Igawa, K. Kodama, S. Shamoto and H. Hosono, Hydrogen in layered iron arsenides: Indirect electron doping to induce superconductivity, Phys. Rev. B 84 (2011) 024521, 1-7.

13. H. Hosono, K. Tanabe, E. Takayama-Muromachi, H. Kageyama, S. Yamanaka, H. Kumakura, M. Nohara, H. Hiramatsu and S. Fujitsu, Exploration of new superconductors and functional materials, and fabrication of superconducting tapes and wires of iron pnictides, Sci. Technol. Adv. Mater. 16 (2015) 033503, 1-87.

14. G. R. Stewart, Superconductivity in iron compounds, Rev. Mod. Phys. 83 (2011) 15891652 .

15. I. I. Mazin, D. J. Singh, M. D. Johannes and M. H. Du, Unconventional superconductivity with a sign reversal in the order parameter of $\mathrm{LaFeAsO}_{1-x} \mathrm{~F}_{x}$, Phys. Rev. Lett. 101 (2008) 057003, 1-4.

16. H. Kontani and S. Onari, Orbital-fluctuation-mediated superconductivity in iron pnictides: Analysis of the five-orbital hubbard-holstein model, Phys. Rev. Lett. 104 (2010) 157001, 1-4.

17. F. Rullier-Albenque, D. Colson, A. Forget and H. Alloul, Multiorbital effects on the transport and the superconducting fluctuations in LiFeAs, Phys. Rev. Lett. 109 (2012) $187005,1-5$.

18. K. Ohgushi and Y. Kiuchi, Doping dependence of Hall coefficient and evolution of coherent electronic state in the normal state of the Fe-based superconductor $\mathrm{Ba}_{1-x} \mathrm{~K}_{x} \mathrm{Fe}_{2} \mathrm{As}_{2}$, Phys. Rev. B $8 \mathbf{5}$ (2012) 064522, 1-5.

19. P. Cheng, H. Yang, Y. Jia, L. Fang, X. Y. Zhu, G. Mu and H. H. Wen, Hall effect and magnetoresistance in single crystals of $\mathrm{NdFeAsO}_{1-x} \mathrm{~F}_{x}(x=0$ and 0.18), Phys. Rev. B 78 (2008) 134508, 1-7.

20. Y. Sun, Y. Ding, B. C. Zheng, Z. X. Shi and Z. A. Ren, Electronic behavior of superconducting $\mathrm{SmFeAsO}_{0.75}$, J. Appl. Phys. 109 (2011) 083914, 1-5.

21. P. Richard, K. Nakayama, T. Sato, M. Neupane, Y. M. Xu, J. H. Bowen, G. F. Chen, J. L. Luo, N. L. Wang, X. Dai, Z. Fang, H. Ding and T. Takahashi, Observation of dirac cone electronic dispersion in $\mathrm{BaFe}_{2} \mathrm{As}_{2}$, Phys. Rev. Lett. 104 (2010) 137001, 1-4. 
22. K. K. Huynh, Y. Tanabe and K. Tanigaki, Both electron and hole dirac cone states in $\mathrm{Ba}(\mathrm{FeAs})_{2}$ confirmed by magnetoresistance, Phys. Rev. Lett. 106 (2011) 217004, 1-4.

23. S. V. Chong, G. V. M. Williams, J. Kennedy, F. Fang, J. L. Tallon and K. Kadowaki, Large low-temperature magnetoresistance in $\mathrm{SrFe}_{2} \mathrm{As}_{2}$ single crystals, Europhys. Lett. 104 (2013) 17002, 1-6.

24. A. O. Sboychakov, A. V. Rozhkov, K. I. Kugel, A. L. Rakhmanov and F. Nori, Electronic phase separation in iron pnictides, Phys. Rev. B 88 (2013) 195142, 1-10.

25. A. Subedi, L. Zhang, D. J. Singh and M. H. Du, Density functional study of FeS, FeSe, and FeTe: Electronic structure, magnetism, phonons, and superconductivity, Phys. Rev. $B \mathbf{7 8}$ (2008) 134514, 1-6.

26. Y. Xia, D. Qian, L. Wray, D. Hsieh, G. F. Chen, J. L. Luo, N. L. Wang and M. Z. Hasan, Fermi surface topology and low-lying quasiparticle dynamics of parent $\mathrm{Fe}_{1+x} \mathrm{Te} / \mathrm{Se}$ superconductor, Phys. Rev. Lett. 103 (2009) 037002, 1-4.

27. F. Chen, B. Zhou, Y. Zhang, J. Wei, H. W. Ou, J. F. Zhao, C. He, Q. Q. Ge, M. Arita, K. Shimada, H. Namatame, M. Taniguchi, Z. Y. Lu, J. Hu, X. Y. Cui and D. L. Feng, Electronic structure of $\mathrm{Fe}_{1.04} \mathrm{Te}_{0.66} \mathrm{Se}_{0.34}$, Phys. Rev. B 81 (2010) 014526, 1-7.

28. Y. Sun, T. Taen, T. Yamada, S. S. Pyon, T. Nishizaki, Z. X. Shi and T. Tamegai, Multiband effects and possible Dirac fermions in $\mathrm{Fe}_{1+y} \mathrm{Te}_{0.6} \mathrm{Se}_{0.4}$, Phys. Rev. B 89 (2014) $144512,1-7$.

29. Y. Tanaka, Multicomponent superconductivity based on multiband superconductors, Supercond. Sci. Technol. 28 (2015) 034002, 1-28.

30. H. Suhl, B. T. Matthias and L. R. Walker, BardeenCooperSchrieffer theory of superconductivity in the case of overlapping bands, Phys. Rev. Lett. 3 (1959) 552-554.

31. J. Bardeen, L. N. Cooper and J. R. Schrieffer, Theory of superconductivity, Phys. Rev. 108 (1957) 1175-1204.

32. J. Kondo, Superconductivity in transition metals, Prog. Theor. Phys. 29 (1963) 1-9.

33. J. Peretti, Superconductivity of transition elements, Phys. Lett. 2 (1962) 275-276.

34. A. J. Leggett, Number-phase fluctuations in two-band superconductors, Prog. Theor. Phys. 36 (1966) 901-930.

35. Y. Tanaka, Phase instability in multi-band superconductors, J. Phys. Soc. Japan 70 (2001) 2844-2847.

36. Y. Tanaka, Soliton in two-band superconductor, Phys. Rev. Lett. 88 (2002) 017002, 1-3.

37. K. H. Ihara, K. Tokiwa, H. Ozawa, M. Hirabayashi, H. Matsuhata, A. Negishi and Y. S. Song, New high- $\mathrm{T}_{c}$ superconductor $\mathrm{Ag}_{1 x} \mathrm{Cu}_{x} \mathrm{Ba}_{2} \mathrm{Ca}_{n 1} \mathrm{Cu}_{n} \mathrm{O}_{2 n+3-\delta}$ family with $\mathrm{T}_{c}>117$, Japan J. Appl. Phys. 33 (1994) L300-L303.

38. N. Hamada and $\mathrm{H}$. Ihara, Electronic band structure of $\mathrm{CuBa}_{2} \mathrm{Ca}_{n-1} \mathrm{Cu}_{n} \mathrm{O}_{2 n+2}$ and $\mathrm{CuBa}_{2} \mathrm{Ca}_{n-1} \mathrm{Cu}_{n} \mathrm{O}_{2 n+1} \mathrm{~F}$ ( $\left.\mathrm{n}=35\right)$, Physica C 357-360 (2001) 108-111.

39. Y. Tanaka, A. Iyo, N. Shirakawa, M. Ariyama, M. Tokumoto, S. I. Ikeda and H. Ihara, Specific heat study on $\mathrm{Cu}_{x} \mathrm{Ba}_{2} \mathrm{Ca}_{n-1} \mathrm{Cu}_{n} \mathrm{O}_{y}$, Physica C 357-360 (2001) 222-225.

40. A. Crisan, Y. Tanaka, D. D. Shivagan, A. Iyo, L. Cosereanu, K. Tokiwa and T. J. Watanabe, Anomalous AC susceptibility response of $(\mathrm{Cu}, \mathrm{C}) \mathrm{Ba}_{2} \mathrm{Ca}_{2} \mathrm{Cu}_{3} \mathrm{O}_{y}$ : Experimental indication of two-component vortex matter in multi-layered cuprate superconductors, Jpn. J. Appl. Phys. 46 (2007) L451-L453.

41. J. Nagamatsu, N. Nakagawa, T. Muranaka, Y. Zenitani and J. Akimitsu, Superconductivity at $39 \mathrm{~K}$ in magnesium diboride, Nature 410 (2001) 63-64.

42. H. Bluhm, N. C. Koshnick, M. E. Huber and K. A. Moler, Magnetic response of mesoscopic superconducting rings with two order parameters, Phys. Rev. Lett. 97 (2006) 237002, 1-4; ibid 98 (2007) 209902(E). 
43. J. W. Guikema, H. Bluhm, D. A. Bonn, R. Liang, W. N. Hardy and K. A. Moler, Twodimensional vortex behavior in highly underdoped $\mathrm{YBa}_{2} \mathrm{Cu}_{3} \mathrm{O}_{6+x}$ observed by scanning Hall probe microscopy, Phys. Rev. B 77 (2008) 104515, 1-10.

44. L. Luan, O. M. Auslaender, D. A. Bonn, R. Liang, W. N. Hardy and K. A. Moler, Magnetic force microscopy study of interlayer kinks in individual vortices in the underdoped cuprate superconductor $\mathrm{YBa}_{2} \mathrm{Cu}_{3} \mathrm{O}_{6+x}$, Phys. Rev. B 79 (2009) 214530, 1-5.

45. Q. Meng, C. N. Varney, H. Fangohr and E. Babaev, Honeycomb, square and kagome vortex lattices in superconducting systems with multiscale intervortex interactions, Phys. Rev. B 90 (2014) 020509(R), 1-5.

46. E. Babaev and M. Speight, Semi-Meissner state and neither type-I nor type-II superconductivity in multicomponent superconductors, Phys. Rev. B 72 (2005) 180502, 1-4.

47. V. Moshchalkov, M. Menghini, T. Nishio, Q. H. Chen, A. V. Silhanek, V. H. Dao, L. F. Chibotaru, N. D. Zhigadlo and J. Karpinski, Type-1.5 superconductivity, Phys. Rev. Lett. 102 (2009) 117001, 1-4.

48. T. Nishio, V. H. Dao, Q. Chen, L. F. Chibotaru, K. Kadowaki and V. V. Moshchalkov, Scanning SQUID microscopy of vortex clusters in multiband superconductors, Phys. Rev. B 81 (2010) 020506, 1-4.

49. E. Babaev, J. Carlström and M. Speight, Type-1.5 superconducting state from an intrinsic proximity effect in two-band superconductors, Phys. Rev. Lett. 105 (2010) 067003, 1-4.

50. J. Gutierrez, B. Raes, A. V. Silhanek, L. J. Li, N. D. Zhigadlo, J. Karpinski, J. Tempere and V. V. Moshchalkov, Scanning Hall probe microscopy of unconventional vortex patterns in the two-gap $\mathrm{MgB}_{2}$ superconductor, Phys. Rev. B 85 (2012) 094511, 1-7.

51. J. Garaud and E. Babaev, Vortex chains due to nonpairwise interactions and fieldinduced phase transitions between states with different broken symmetry in superconductors with competing order parameters, Phys. Rev. B 91 (2015) 014510, 1-8.

52. E. Y. Vedmedenko, Competing Interactions and Patterns in Nanoworld (Wiley-VCH Verlag GmbH \& Co. KGaA, 2007).

53. P. Ball, The Self-Made Tapestry: Pattern Formation in Nature (Oxford Univ. Press, Oxford, U.K., 1999).

54. F. Nori, Intermittently flowing rivers of quantized magnetic flux, Science $\mathbf{2 7 1}$ (1996) 1373-1374.

55. C. J. Olson, C. Reichhardt and F. Nori, Superconducting vortex avalanches, voltage bursts, and vortex plastic flow: Effect of the microscopic pinning landscape on the macroscopic properties, Phys. Rev. B 56 (1997) 6175-6194.

56. C. J. Olson, C. Reichhardt and F. Nori, Fractal networks, braiding channels and voltage noise in intermittently flowing rivers of quantized magnetic flux, Phys. Rev. Lett 80 (1998) 2197-2200.

57. A. P. Mehta, C. Reichhardt, C. J. Olson and F. Nori, Topological invariants in microscopic transport on rough landscapes: Morphology and Horton analysis of river-like networks of vortices, Phys. Rev. Lett. 82 (1999) 3641-3644.

58. M. F. Laguna, C. A. Balseiro, D. Domínguez and F. Nori, Vortex structure and dynamics in kagomé and triangular, Phys. Rev. B 64 (2001) 104505, 1-13.

59. H. J. Zhao, V. R. Misko and F. M. Peeters, Analysis of pattern formation in systems with competing range interactions, New J. Phys. 14 (2012) 063032, 1-21.

60. H. J. Zhao, V. R. Misko and F. M. Peeters, Dynamics of self-organized driven particles with competing range interaction, Phys. Rev. E 88 (2013) 022914, 1-7.

61. H. J. Zhao, V. R. Misko, J. Tempere and F. Nori, Pattern formation in vortex matter with pinning and frustrated intervortex interactions, Phys. Rev. B 95 (2017) 104519, 1-9. 
62. P. J. Curran, W. M. Desoky, M. V. Milosevic, A. Chaves, J.-B. Laloë, J. S. Moodera and S. J. Bending, Spontaneous symmetry breaking in vortex systems with two repulsive lengthscales, Sci. Rep. 5 (2015) 15569, 1-9.

63. X. B. Xu, H. Fangohr, M. Gu, W. Chen, Z. H. Wang, F. Zhou, D. Q. Shi and S. X. Dou, Simulation of the phase diagram of magnetic vortices in two-dimensional superconductors: Evidence for vortex chain formation, J. Phys.: Condens. Matter 26 (2014) $115702,1-6$.

64. J. Van de Vondel, V. N. Gladilin, A. V. Silhanek, W. Gillijns, J. Tempere, J. T. Devreese and V. V. Moshchalkov, Vortex core deformation and stepper-motor ratchet behavior in a superconducting aluminum film containing an array of holes, Phys. Rev. Lett. 106 (2011) 137003, 1-4.

65. J. Tempere, E. Vermeyen and B. Van Duppen, Skyrmion rows, vortex rows, and phase slip lines in sheared multi-component condensates, Physica C 479 (2012) 61-64.

66. I. Bloch, J. Dalibard and W. Zwerger, Many-body physics with ultracold gases, Rev. Mod. Phys. 80 (2008) 885-964.

67. J. Tempere, S. Ceuppens and E. Vermeyen, Composite vortices in two-component BoseEinstein condensates, J. Phys: Conf. Series 414 (2013) 012035, 1-7.

68. J. Gutiérrez, A. Llordés, J. Gázquez, M. Gibert, N. Romà, S. Ricart, A. Pomar, F. Sandiumenge, N. Mestres, T. Puig and X. Obradors, Strong isotropic flux pinning in solution-derived $\mathrm{YBa}_{2} \mathrm{Cu}_{3} \mathrm{O}_{7-x}$ nanocomposite superconductor films, Nature Mat. 6 (2006) 367-373.

69. V. V. Moshchalkov, M. Baert, V. V. Metlushko, E. Rosseel, M. J. Van Bael, K. Temst, R. Jonckheere and Y. Bruynseraede, Magnetization of multiple-quanta vortex lattices, Phys. Rev. B 54 (1996) 7385-7393.

70. J. E. Villegas, S. Savel'ev, F. Nori, E. M. Gonzalez, J. V. Anguita, R. García and J. L. Vicent, A superconducting reversible rectifier that controls the motion of magnetic flux quanta, Science 302 (2003) 1188-1191.

71. R. Wördenweber, P. Dymashevski and V. R. Misko, Guidance of vortices and the vortex ratchet effect in high- $\mathrm{T}_{c}$ superconducting thin films obtained by arrangement of antidots, Phys. Rev. B 69 (2004) 184504, 1-6.

72. V. R. Misko, S. Savel'ev, A. L. Rakhmanov and F. Nori, Nonuniform self-organized dynamical states in superconductors with periodic pinning, Phys. Rev. Lett. 96 (2006) 127004 1-4; ibid Negative differential resistivity in superconductors with periodic arrays of pinning sites, Phys. Rev. B 75 (2007) 024509 1-10.

73. $\mathrm{P}$. Selders and R. Wördenweber, Low-frequency noise reduction in $\mathrm{YBa}_{2} \mathrm{Cu}_{3} \mathrm{O}_{7-\delta}$ $\mathrm{YBa}_{2} \mathrm{Cu}_{3} \mathrm{O}_{7-\delta}$ superconducting quantum interference devices by antidots, Appl. Phys. Lett. 76 (2000) 3277-3279.

74. P. Lahl and R. Wördenweber, Probing microwave properties of high- $\mathrm{T}_{c}$ films via small dc magnetic fields, Appl. Phys. Lett. 81 (2002) 505-507.

75. P. Bushev, D. Bothner, J. Nagel, M. Kemmler, K. B. Konovalenko, A. Loerincz, K. Ilin, M. Siegel, D. Koelle, R. Kleiner and F. Schmidt-Kaler, Trapped electron coupled to superconducting devices, Eur. Phys. J. D 63 (2011) 9-16.

76. V. Misko, S. Savel'ev and F. Nori, Critical currents in quasiperiodic pinning arrays: Chains and penrose lattices, Phys. Rev. Lett. 95 (2005) 177007, 1-4.

77. V. R. Misko, S. Savel'ev and F. Nori, Critical currents in superconductors with quasiperiodic pinning arrays: One-dimensional chains and two-dimensional Penrose lattices, Phys. Rev. B $\mathbf{7 4}$ (2006) 024522, 1-20.

78. J. E. Villegas, M. I. Montero, C.-P. Li and I. K. Schuller, Correlation length of quasiperiodic vortex lattices, Phys. Rev. Lett. 97 (2006) 027002, 1-4. 
79. M. Kemmler, C. Gürlich, A. Sterck, H. Pöhler, M. Neuhaus, M. Siegel, R. Kleiner and D. Koelle, Commensurability effects in superconducting $\mathrm{Nb}$ films with quasiperiodic pinning arrays, Phys. Rev. Lett. 97 (2006) 147003, 1-4.

80. A. V. Silhanek, W. Gillijns, V. V. Moshchalkov, B. Y. Zhu, J. Moonens and L. H. A. Leunissen, Enhanced pinning and proliferation of matching effects in a superconducting film with a Penrose array of magnetic dots, Appl. Phys. Lett. 89 (2006) 152507, 1-3.

81. R. B. G. Kramer, A. V. Silhanek, J. Van de Vondel, B. Raes and V. V. Moshchalkov, Symmetry-induced giant vortex state in a superconducting $\mathrm{Pb}$ film with a fivefold Penrose array of magnetic pinning centers, Phys. Rev. Lett. 103 (2009) 067007, 1-4.

82. R. Penrose, Pentaplexity a class of non-periodic tilings of the plane, Math. Intelligencer 2(1) (1979) 32-37.

83. D. Levine and P. J. Steinhardt, Quasicrystals: A new class of ordered structures, Phys. Rev. Lett. 53 (1984) 2477-2480.

84. H. C. Jeong and P. J. Steinhardt, Constructing Penrose-like tilings from a single prototile and the implications for quasicrystals, Phys. Rev. B 55 (1997) 3520-3532.

85. P. J. Steinhardt, H.-C. Jeong, K. Saitoh, M. Tanaka, E. Abe and A. P. Tsai, Experimental verification of the quasi-unit-cell model of quasicrystal structure, Nature (London) 396 (1998) 55-57.

86. V. R. Misko, D. Bothner, M. Kemmler, R. Kleiner, D. Koelle, F. M. Peeters and F. Nori, Enhancing the critical current in quasiperiodic pinning arrays below and above the matching magnetic flux, Phys. Rev. B 82 (2010) 184512, 1-7.

87. D. Bothner, R. Seidl, V. R. Misko, R. Kleiner, D. Koelle and M. Kemmler, Unusual commensurability effects in quasiperiodic pinning arrays induced by local inhomogeneities of the pinning site density, Supercond. Sci. Technol. 27 (2014) 065002, 1-8.

88. T. Frankel, The Geometry of Physics: An Introduction, 2nd ed. (Cambridge University Press, Cambridge, UK, 2004).

89. David R. Nelson, Defects $\&$ Geometry in Condensed Matter Physics, 2nd ed. (Cambridge University Press, Cambridge, UK, 2002).

90. J.-F. Sadoc and R. Mosseri, Geometrical Frustration (Cambridge University Press, Cambridge, UK, 1999).

91. M. J. Bowick and L. Giomi, Two-dimensional matter: Order, curvature and defects, Adv. Phys. 58 (2009) 449-563.

92. D. R. Nelson, Order, frustration, and defects in liquids and glasses, Phys. Rev. B 28 (1983) 5515-5535.

93. D. R. Nelson and M. Widom, Symmetry, Landau theory and polytope models of glass, Nucl. Phys. B 240 (1984) 113-139.

94. P. J. Steinhardt, D. R. Nelson and M. Ronchetti, Icosahedral bond orientational order in supercooled liquids, Phys. Rev. Lett. 47 (1981) 1297-1300; ibid Bond-orientational order in liquids and glasses, Phys. Rev. B 28 (1983) 784-805.

95. A. M. Turner, V. Vitelli and D. R. Nelson, Vortices on curved surfaces, Rev. Mod. Phys. 82 (2010) 1301-1348.

96. I. V. Grigorieva, W. Escoffier, J. Richardson, L. Y. Vinnikov, S. Dubonos and V. Oboznov, Direct observation of vortex shells and magic numbers in mesoscopic superconducting disks, Phys. Rev. Lett. 96 (2006) 077005, 1-4.

97. I. V. Grigorieva, W. Escoffier, V. R. Misko, B. J. Baelus, F. M. Peeters, L. Y. Vinnikov and S. V. Dubonos, Pinning-induced formation of vortex clusters and giant vortices in mesoscopic superconducting disks, Phys. Rev. Lett. 99 (2007) 147003, 1-4.

98. V. A. Schweigert, F. M. Peeters and P. S. Deo, Vortex phase diagram for mesoscopic superconducting disks, Phys. Rev. Lett. 81 (1998) 2783-2786. 
99. A. Kanda, B. J. Baelus, F. M. Peeters, K. Kadowaki and Y. Ootuka, Experimental evidence for giant vortex states in a mesoscopic superconducting disk, Phys. Rev. Lett. 93 (2004) 257002, 1-4.

100. L. F. Chibotaru, A. Ceulemans, V. Bruyndoncx and V. V. Moshchalkov, Symmetryinduced formation of antivortices in mesoscopic superconductors, Nature (London) 408 (2000) 833-835; ibid Vortex entry and nucleation of antivortices in a mesoscopic superconducting triangle, Phys. Rev. Lett. 86 (2001) 1323-1326.

101. V. R. Misko, V. M. Fomin, J. T. Devreese and V. V. Moshchalkov, Stable vortexantivortex molecules in mesoscopic superconducting triangles, Phys. Rev. Lett. 90 (2003) 147003, 1-4.

102. V. R. Misko and F. Nori, Magnetic flux pinning in superconductors with hyperbolictessellation arrays of pinning sites, Phys. Rev. B 85 (2012) 184506, 1-6.

103. D. Ray, C. J. Olson Reichhardt, B. Jankó and C. Reichhardt, Strongly enhanced pinning of magnetic vortices in type-II superconductors by conformal crystal arrays, Phys. Rev. Lett. 110 (2013) 267001, 1-5.

104. D. Ray, C. Reichhardt and C. J. Olson Reichhardt, Pinning, ordering, and dynamics of vortices in conformal crystal and gradient pinning arrays, Phys. Rev. B 90 (2014) 094502, 1-16.

105. C. Reichhardt, D. Ray and C. J. Olson Reichhardt, Reversible ratchet effects for vortices in conformal pinning arrays, Phys. Rev. B 91 (2015) 184502, 1-9.

106. M. Motta, F. Colauto, W. A. Ortiz, J. Fritzsche, J. Cuppens, W. Gillijns, V. V. Moshchalkov, T. H. Johansen, A. Sanchez and A. V. Silhanek, Enhanced pinning in superconducting thin films with graded pinning landscapes, Appl. Phys. Lett. 102 (2013) 212601, 1-4.

107. Y. L. Wang, M. L. Latimer, Z. L. Xiao, R. Divan, L. E. Ocola, G. W. Crabtree and W. K. Kwok, Enhancing the critical current of a superconducting film in a wide range of magnetic fields with a conformal array of nanoscale holes, Phys. Rev. B 87 (2013) 220501(R), 1-5.

108. S. Guénon, Y. J. Rosen, Ali C. Basaran and Ivan K. Schuller, Highly effective superconducting vortex pinning in conformal crystals, Appl. Phys. Lett. 102 (2013) 252602, $1-4$.

109. J. George, A. Jones, M. Al-Qurainy, S. A. Fedoseev, A. Rosenfeld and A. V. Pan, Tunable pinning effects produced by non-uniform antidot arrays in YBCO thin films, Ann. Phys. (Berlin) 529 (2017) 1600283, 1-7. 